\title{
METEORITOS Y METEOROS EN COSTA RICA (VERDADEROS, POSIBLES Y FALSOS)
}

\author{
Gerardo J. Soto \\ Consultor, Apdo. 360-2350 San Francisco de Dos Ríos, Costa Rica \\ correo-e: katomirodriguez@yahoo.com
}

(Recibido 12/1/05; Aceptado 14/2/05)

\begin{abstract}
Geological, documentary (since 1799) and anecdotal (second half of the 20th century) information on meteorites and meteors in Costa Rica has been compiled. There is only one case of sighting and recovering a meteorite, in Heredia on April $1^{\text {st }}$, 1857, from which a fragment $(2.9 \mathrm{~g})$ is preserved in Costa Rica. It is an H5 chondrite with olivine, bronzite and metallic minerals, probably originated in the asteroid 6 Hebe. Two more meteorites would have landed in 1912 and 1962 or 63, though we lack of the rock proof. There are also three documented cases of fireballs, two false fireballs (cases of anthropogenic bodies), four doubtful cases (possible anthropogenic bodies), and two supposed impact craters that resulted not to be true. According to world extrapolations, probabilities that a meteorite weighting $>1 \mathrm{~kg}$ falls in Costa Rica, are $0.44 /$ year, despite it would be expectable to recover a meteorite in Costa Rica every 1200 years of history. The legal deficiencies about the property of meteorite falls and insurance against meteorites are discussed.
\end{abstract}

Keywords: Costa Rican meteorites, meteors, Heredia chondrite, meteorite fall probabilities, property, insurance.

RESUMEN: Se ha recabado información geológica, documental (desde 1799) y anecdótica (segunda mitad del siglo XX) sobre meteoritos y meteoros en Costa Rica. Un único caso de avistamiento y recuperación de un meteorito se dio en Heredia el $1^{\circ}$ de abril de 1857, del cual se conserva un fragmento de $2,9 \mathrm{~g}$ en Costa Rica. Se trata de un condrito H5 con olivino, broncita y minerales metálicos, probablemente originado en el asteroide 6 Hebe. Dos meteoritos más habrían caído en 1912 y 1962 ó 63, pero se carece de las pruebas rocosas. Hay tres casos documentados de bolas de fuego, dos falsas bolas de fuego (casos de cuerpos antropogénicos), cuatro casos incógnitos (posibles cuerpos antropogénicos), y dos supuestos cráteres de impacto que resultaron no serlo. Con base en extrapolaciones mundiales, las probabilidades de que caiga un meteorito en Costa Rica, con un peso de al menos $1 \mathrm{~kg}$ son de 0,44/año, aunque sería esperable recuperar un meteorito cada 1200 años de historia. Se discuten las lagunas legales que existen sobre la propiedad de meteoritos caídos y seguros contra meteoritos.

Palabras clave: Meteoritos de Costa Rica, meteoros, condrito Heredia, probabilidades de caída meteorítica, propiedad, seguros. 


\section{INTRODUCCIÓN}

A pesar de que el estudio científico de los meteoritos data desde el trabajo pionero de Ernst Chladni en 1794, hasta hace unas cuatro décadas, los meteoritos eran un grupo de rarezas estudiadas por unos cuantos científicos cuasiesotéricos, con tal de conocer de una manera directa, la más asequible, la composición de los planetas del Sistema Solar. Aparecieron luego, trabajos auténticamente seminales (verbigracia Mason, 1962), que marcaron un hito, tanto por su exposición para los especialistas, como para los legos. En 1963 vieron la luz varias publicaciones científicas que trataban sobre el famoso Cráter del Meteoro, en Arizona, y sobre las estructuras circulares, que discutían si eran volcánicas o el producto de impactos de meteoritos (un completo recuento histórico se encuentra en French, 1990). También, algún tiempo atrás se pensó que los cráteres de la Luna y de los otros planetas terrestres eran de origen volcánico, hasta que se tuvo la certeza de su origen por impactos meteoríticos, a lo cual cooperó, con mucho, el muestrario de rocas lunares traídas por las misiones Apolo.

La gran revolución en el estudio de los meteoritos la causó, al inicio de los años ochenta, la hipótesis de Alvarez et al. (1980), de que la gran extinción masiva a finales del Cretácico, hace 65 $\mathrm{Ma}$, fue causada por un gran impacto de un meteorito que lanzó billones de toneladas de polvo terrestre y extraterrestre hacia la atmósfera y causó un invierno letal, incluso para los dinosaurios. Diez años después, se supo que el lugar más probable de ese impacto fue en la actual costa de Yucatán, cerca del poblado de Chicxulub, causado por un meteorito que ha de haber tenido unos $10 \mathrm{~km}$ de diámetro (Hildebrand et al., 1991). Sin embargo, estas catátrofes son escasas. Son más comunes los visitantes pétreos de tamaño más moderado, de apenas unos metros o decámetros. Caen de vez en cuando, y muchos de ellos no llegan a tocar la superficie terrestre, sino que se consumen en su ingreso a la atmósfera.

En este ensayo se repasan algunos casos conocidos de meteoros, bolas de fuego, meteoritos, impactos, falsos meteoritos e incógnitas, documentados histórica y anecdóticamente en
Costa Rica. No es absolutamente exhaustivo, por lo que queda pendiente la tarea de ampliar el listado aquí mostrado.

\section{Meteoroides, meteoros y meteoritos: cuerpos extraterrestres}

Vagando a través del Sistema Solar en órbitas elípticas, están los meteoroides, con tamaños que oscilan entre unos $10 \mathrm{~km}$ a menos de un $\mathrm{mm}$, productos de colisiones entre asteroides o cometas desintegrados. Cuando se aproximan a la Tierra y son atraídos por su fuerza gravitacional, entran a la atmósfera, donde la fricción los calienta hasta la incandescencia, y se les llama meteoros (conocidos como "estrellas fugaces"). A los más brillantes, que dejan una estela en el cielo, se les llama bolas de fuego, y son los que alcanzan tamaños más grandes que un guijarro.

Mientras los meteoroides pasan por la atmósfera, se vaporizan, funden y fragmentan, y si no son consumidos totalmente en su ingreso al planeta, llegan a la superficie: estos son los meteoritos. Algunos, como el mencionado de Chicxulub, son enormes moles, capaces de causar una gran catástrofe. Solo los más grandes y compactos meteoroides tocan la superficie como meteoritos a gran velocidad. Muchos otros son microscópicos -micrometeoritos-, que descienden a baja velocidad por el roce con el aire, para depositarse finalmente en tierra o en el fondo oceánico.

Hay tres clases de meteoritos: los férreos, compuestos principalmente de hierro y níquel, muy densos; los pétreos, los más abundantes, compuestos de silicatos densos de hierro y magnesio, y los férro-pétreos, intermedios entre los anteriores.

Los asteroides parentales de los meteoritos se formaron como cuerpos kilométricos con núcleo metálico y el resto silicático, que formaron protoplanetas errantes al principio del Sistema Solar, y permanecen, aunque fragmentados. Tales cuerpos nos han dado la edad del Sistema: se enfriaron hace 4560 millones de años (detalladas dataciones radiométricas en Hutchison, 1992, y sobre los cuerpos parentales, 
en McSween, 1999). Debido a que los meteoritos contienen materiales del Sistema Solar primigenio, son fuente importante de información sobre el origen y evolución de la materia cósmica. Su singularidad radica en que son cuerpos extraterrestres, la mayoría son los más viejos y primitivos del Sistema, y algunos provienen de la Luna y Marte. Algunos de ellos además, han sido tocados por polvo estelar proveniente de otras estrellas o sistemas solares.

Puesto que los mares son tres cuartas partes de la superficie terrestre, y si de la superficie continental sustraemos los desiertos fríos y calientes, es evidente el porqué los meteoritos avistados y recuperados al año en el mundo, son contables con los dedos de una mano. No es fácil encontrar meteoritos. Como ejemplo China, un país de $10^{7} \mathrm{~km}^{2}$ y larga historia documentada ( 4000 años), tiene uno de los registros de meteoritos más documentados: cerca de 700 . El más antiguo relato de una caída meteorítica, en el año 645 a.C. proviene de China, así como la documentación del uso de un meteorito férrico en una hoja de un arma del siglo XIII a.C. (Lin et al., 1995). Si extrapolamos la extensión, la historia y la cantidad de meteoritos hallados en China, y la comparamos con Costa Rica, se infiere que se podría recuperar un meteorito en Costa Rica cada 1200 años de historia. De hecho, en América Central sólo hay tres meteoritos documentados como vistos caer y recuperados (Graham et al., 1985): el llamado "Rosario", encontrado en 1896 en Honduras, un meteorito férrico (octaedrito con grandes bandas de Widmannstätten) del cual existen muestras en Nueva York (2 kg), en el Museo Británico (118 g) y en el Museo de París (200 g); el "Chinautla", recuperado en 1902 en Guatemala, que también es férrico (un octaedrito medio con una masa de 5,7 kg), de los cuales, 1200 g están en el Museo Británico y 133 g en París, y el tercero es el meteorito Heredia, caído en Costa Rica, del que se dan detalles en este trabajo. Por otra parte, una vistosa bola de fuego se vio a lo largo de gran parte de Nicaragua el 26 de abril de 1997, erróneamente reportada como un meteorito, pues no hubo recuperación de cuerpo (detalles se pueden encontrar en Strauch, 1997).

\section{POSIBLES METEORITOS DEL PASADO EN COSTA RICA}

En los últimos miles de años, es seguro que hayan caído meteoritos en el territorio costarricense. El problema es encontrarlos o identificarlos. En la Luna sin atmósfera, o en Antártida cubierta de nieve, seca y fría, los meteoritos se conservan inalterados, prístinos, y son fáciles de divisar. En cambio, con la abundante vegetación en sitios tropicales, las posibilidades de distinguir meteoritos en el terreno, son bajas. Además, la humedad ambiental y del suelo, degradan aceleradamente los meteoritos, por sus componentes metálicos y silicáticos. Por ambas razones, son casi inencontrables en Costa Rica. Nos queda, sin embargo, una huella por seguir como documento de caídas meteoríticas pasadas: los cráteres de impacto. Se analizan dos casos que se propusieron y resultaron no ser cráteres de impacto (Cuadro 1; Fig. 1). Algunas formas circulares de hasta un kilómetro de diámetro (como el Bajo de la Rosa, al noroeste del volcán Irazú), han sido interpretadas como cráteres, ya fuera de impacto o volcánico, pero suelen resultar geoformas de deslizamiento y erosión, bastante frecuentes en Costa Rica, pero no relacionadas con impactos.

\section{El falso cráter meteorítico de la laguna de Río Cuarto}

La laguna de Río Cuarto se localiza cerca del poblado homónimo, entre San Miguel de Sarapiquí y Aguas Zarcas (Fig. 1), en el inicio de las llanuras del norte del país. Su forma es casi circular y tiene unas 40 hectáreas de superficie, con paredes internas de fuerte pendiente, que bajan entre 5 y $20 \mathrm{~m}$ entre el borde y el nivel de las aguas. A pesar de que don Anastasio Alfaro (1924) había escrito que esta oquedad era de origen volcánico, en 1978 la revista Gentes y Paisajes (Salguero, 1978) publicó un reportaje en el que hacía eco de la hipótesis lanzada por la Motorola Aerial Sensing Inc., cuyos personeros, basados en imágenes de radar, supusieron que el hoyo ocupado por la laguna, fuese un cráter de impacto. 


\section{Cuadro 1}

Ubicación de meteoritos, bolas de fuego, falsas bolas de fuego, falsos cráteres de impacto e incógnitas en Costa Rica

\begin{tabular}{|c|c|c|c|}
\hline $\mathrm{N}^{\circ}$ Fig. 1 & Evento & Latitud N & Longitud W \\
\hline \multicolumn{4}{|l|}{ Meteoritos } \\
\hline 1 & Heredia 1857 & 10,000 & 84,120 \\
\hline 2 & San Pedro de Poás 1962-63? & 10,083 & 84,248 \\
\hline 3 & Tortuguero (dudoso) 1912 & 10,516 & 83,710 \\
\hline \multicolumn{4}{|c|}{ Bolas de fuego } \\
\hline 4 & Cartago 1910 & 10,000 & Entre 83,750 y 86,000 \\
\hline 5 & Miramar 1991 & 10,096 & 84,731 \\
\hline 6 & Punta Mala 1999 & 09,058 & 83,631 \\
\hline \multicolumn{4}{|c|}{ Falsas bolas de fuego } \\
\hline 7 & Limón 1990 & 10,000 & 83,028 \\
\hline 8 & Florencia 1994 & 10,364 & 84,477 \\
\hline \multicolumn{4}{|c|}{ Falsos cráteres de impacto } \\
\hline 9 & Río Cuarto & 10,358 & 84,219 \\
\hline 10 & Savegre & 09,450 & 83,959 \\
\hline \multicolumn{4}{|l|}{ Incógnitas } \\
\hline 11 & Barva 1905 & 10,023 & 84,126 \\
\hline 12 & La Cruz 1993 & 11,020 & 85,624 \\
\hline 13 & Lomas de Barbudal 1994 & 10,483 & 85,392 \\
\hline 14 & Río Tulín 1995 & 09,666 & 84,500 \\
\hline
\end{tabular}

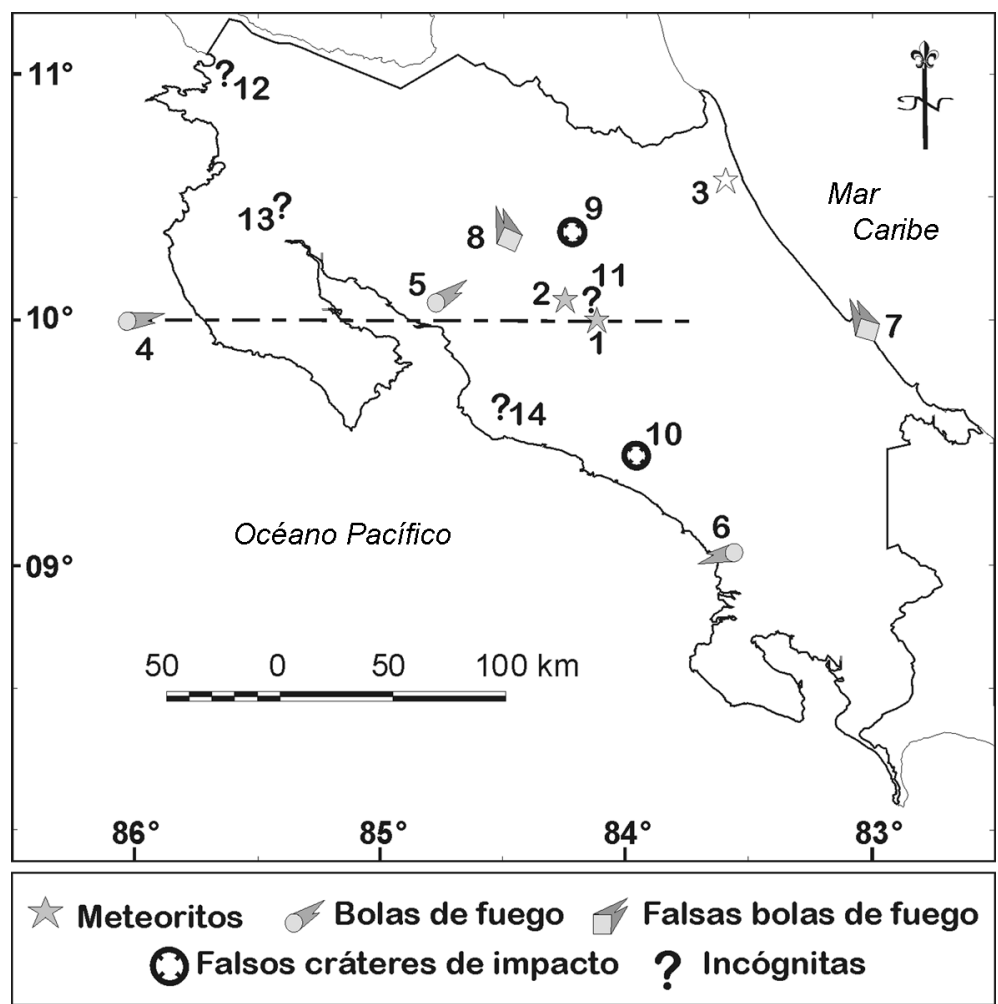

Fig. 1: Ubicación de meteoritos, bolas de fuego, falsas bolas de fuego, falsos cráteres de impacto e incógnitas en Costa Rica (números y detalles según el Cuadro 1). 
Empero, un análisis más detallado, tanto geomorfológico como geovulcanológico (véase por ejemplo Alvarado, 1989) ha permitido corroborar el origen de este paisaje por un evento volcánico de explosión, que desecha totalmente la hipótesis de cráter por impacto meteorítico.

\section{El supuesto cráter de impacto de Savegre}

En un mapa geológico-fotogeológico con fines hidroeléctricos, de la cuenca del río Savegre (Dengo \& Escalante, 1980), sobresalía una estructura de $600 \mathrm{~m}$ de diámetro, sospechosamente circular cerca de la confluencia de los ríos Savegre y División (Fig. 1), sobre rocas sedimentarias de edad terciaria, muy cerca de una falla, por lo que los autores sugirieron que podría ser un cráter por impacto de meteorito. Una visita posterior de algunos geólogos del Instituto Costarricense de Electricidad (ICE), desechó la teoría del cráter de impacto, y más bien lo catalogaron como una cárcava de erosión (S. Mora, com. oral, 1995). Parece muy improbable que se preserve una estructura de este tipo, de tamaño tan modesto, en un ambiente tan agreste como el de esa zona, por lo que la explicación última es plausible.

\section{DOCUMENTOS HISTÓRICOS}

\section{Las Leónidas (lluvia de meteoros) de noviembre de 1799}

Cleto González Víquez reprodujo en su libro sobre eventos naturales en Costa Rica (González, 1910), una nota dirigida al Capitán General en Guatemala, por el entonces gobernador Tomás de Acosta, fechada el 20 de noviembre de 1799 , sobre una lluvia de meteoros acaecida en la noche entre el 11 y 12 de noviembre. La descripción de Acosta parece bastante exagerada, en tanto que usa hipérboles como: "Muchas eran de tal magnitud que parecían globos de fuego, ó carcaces encendidos, y arrojaban tan copiosas y gruesas chispas que caían algunas hasta el suelo...", “...sólo he sabido de una que cayó en el pueblo de los indios de Tobosi, como á una legua de esta ciudad, la cual dicen estos ardió largo rato en gruesa llama y á proporción que esta disminuía tomaba un color azul...”, “...la expresión de que llovió fuego sólo se aparta de la realidad en que no cayó en tierra todo el que se desprendió de la atmósfera". Don Cleto acota que "el fenómeno lo presenció Humboldt desde Cumaná y del cual publica Flammarión en su Astronomía de las Damas el grabado ó dibujo del navegante inglés Ellicott, testigo presencial".

En efecto, Humboldt y Bonpland (este último "que se había levantado para gozar del fresco en la galería los percibió primero" a las 2 ? de la mañana: Humboldt, 1941, Tomo II, p. 230) observaron la lluvia de meteoros estando en Cumaná, Colombia, y luego realizaron una minuciosa investigación del fenómeno, preguntando dondequiera que fueron en Sudamérica. Acota Humboldt que "Cuál no sería mi admiración cuando al volver a Europa supe que el mismo fenómeno había sido reparado en una extensión del globo de $64^{\circ}$ de latitud y $91^{\circ}$ de longitud, en el ecuador, en la América meridional, en el Labrador y en Alemania!" (Humboldt, 1941, Tomo II, p. 234). Posteriormente, en su obra Cosmos (Humboldt, 1844), dio una amplia explicación científica al fenómeno, asociándolo con las estrellas fugaces y los meteoritos.

Esta lluvia de meteoros ocurre a mediados de noviembre cada año, y es conocida hoy como "Las Leónidas", porque parece radiar de la constelación de Leo. Los eventos más brillantes se suceden cada 33 a 34 años (por ejemplo Humboldt había anotado que en 1866 la habían visto en Cumaná y en Quito). Una de las más notables ocurrió en 1966, cuando se pudieron observar hasta 19000 meteoros por hora en el suroeste de Estados Unidos. En el 2001, también importante, el pico llegó a 8000 meteoros por hora contados en el sur de Japón. Por la posición de la Tierra, no todos los puntos del planeta tienen la misma visión cada año. El primero de que se tiene noticia ocurrió en el año 585 d.C., aunque los más significativos empezaron en el siglo IX y no habrán más después del año 2164 (un profuso detalle de Las Leónidas se puede encontrar en Littmann, 1998). 


\section{El meteorito Heredia: $1^{0}$ de abril de 1857}

Entre las 7 y 8 de la noche del $1^{\circ}$ de abril de 1857, la población del Valle Central de Costa Rica fue sorprendida por una bola de fuego, que cruzó San José y causó un impacto detonante en Heredia (Fig. 1). Una reseña de este evento se encuentra en el libro de Don Cleto (González, 1910), y algunas acotaciones adicionales fueron hechas por Soto (1989a y b).

El periódico La Crónica de Costa Rica del 4 de abril describió que "la Capital se vio repentinamente iluminada por un meteoro que cruzó de Sur á Norte lanzando grandes exhalaciones". El mismo periódico refiere (en su edición del 9 de mayo) que "el meteoro... lanzó....unas grandes piedras negruzcas, metálicas y como de unas cinco o seis libras de peso, que en vano hemos querido analizar". El aspecto negruzco exterior es común en estos cuerpos, pues se trata de una corteza generada por fusión de la zona externa a causa del calor desarrollado durante la fricción con la atmósfera.

Domeyko (1859) describió por primera vez las muestras obtenidas, reportando que éstas eran varias, la mayor de más de $1 \mathrm{~kg}$ de peso. El meteorito apareció nominado en el listado Büchner (1863) como "Heredia", debido al lugar de su impacto. Luego fue estudiado un poco más en detalle por Mason (1963), quien lo catalogó como "uno del tipo H5, una condrita olivino-broncítica brechiada, con $18 \%$ de olivino". Las condritas pertenecen al grupo de los meteoritos pétreos y son los más antiguos y casi inalterados a través de toda la historia del Sistema Solar. Se les llama así porque tienen cóndrulos, que son esferitas de silicatos de tamaño milimétrico. Las condritas del tipo $\mathrm{H}$ son las que tienen la más alta cantidad de hierro, presente en el olivino, la broncita y en parte en estado metálico (Fig. 2). Los números que siguen a la $\mathrm{H}$ son una clasificación petrológica que indica el grado de alteración de los cóndrulos por efecto del calentamiento a lo largo de su historia. El número 5 indica un grado alto de metamorfismo por calor, que hace los cóndrulos menos distinguibles. Schultz \& Kruse (1983) lo catalogaron como un meteorito rico en gases. Con base en las características físicas observadas

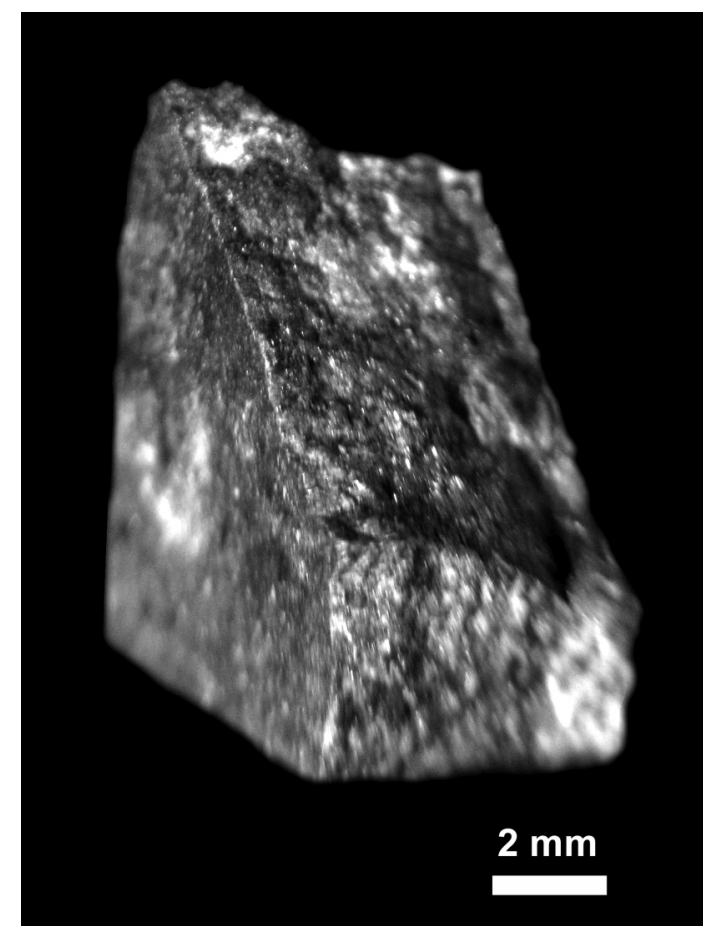

Fig. 2: Fotografía del espécimen del meteorito Heredia exhibido en la Escuela Centroamericana de Geología de la Universidad de Costa Rica. Las áreas brillantes en la parte izquierda, arriba de la cara frontal superior y del lado derecho de la cara frontal inferior corresponden con minerales metálicos.

por los astrónomos en los asteroides y su comparación con las de los meteoritos, se ha podido especular cuáles asteroides son los cuerpos de origen de los diferentes tipos de meteoritos. Los de tipo $\mathrm{H}$, con alta probabilidad proceden del asteroide 6 Hebe, que tiene 185 kilómetros de diámetro y está en el cinturón principal de asteroides (véanse detalles de los cuerpos de origen de los meteoritos en McSween, 1999). De tal manera que el meteorito Heredia, muy probablemente procede de ese asteroide.

Aunque en Costa Rica no se reportaba que hayan quedado muestras del cuerpo extraterrestre, hasta el año 1999 había quince institutos en el mundo que guardaban fragmentos de él y cinco con secciones delgadas (Cuadro 2). Algunos otros fragmentos en colecciones privadas no son conocidos, pero probablemente existen. 
Cuadro 2

Instituciones del mundo con muestras del meteorito Heredia

\begin{tabular}{llr}
\hline $\mathrm{N}^{\circ}$ & Institución, ciudad, país & Peso (g) \\
\hline 1 & Instituto de Mineralogía, Universidad de Gotinga, Alemania & 410,0 \\
2 & Escuela de Minas de París, Francia & 80,0 \\
3 & Instituto de Mineralogía y Petrografía, Universidad de Tubinga, Alemania & 68,9 \\
4 & Museo de Historia Natural de Londres, Inglaterra & 52,5 \\
5 & Museo de Historia Natural de París, Francia & 37,5 \\
6 & Colección DuPont, Nueva Jersey, EE.UU. & 33,0 \\
7 & Museo de Historia Natural de Viena, Austria & 24,0 \\
8 & Museo Field de Historia Natural de Chicago, EE.UU. & 9,5 \\
9 & Museo Estadounidense de Historia Natural de Nueva York, EE.UU. & 7,5 \\
10 & Observatorio Tiara, Colorado Springs, EE.UU. & 5,7 \\
11 & Colección del Observatorio Vaticano, Roma & 5,0 \\
11 & Universidad Estatal de Arizona, en Tempe, EE.UU. & 4,0 \\
13 & Museo de Historia Natural de la Universidad Humboldt, Berlín, Alemania & 2,4 \\
14 & Museo del Servicio Geológico de Canadá, Ottawa & 2,0 \\
15 & Museo del Servicio Geológico de la India, Calcuta & 1,0 \\
& Secciones delgadas & - \\
1 & Museo de Historia Natural de la Universidad Humboldt, Berlín, Alemania & - \\
3 & Instituto de Mineralogía, Universidad de Gotinga, Alemania & - \\
4 & Museo de Historia Natural de Londres, Inglaterra & - \\
5 & Museo de Historia Natural de París, Francia & - \\
\hline
\end{tabular}

En marzo de 1999, la New England Meteoritical Services (NEMS) listó en su catálogo de ventas una pequeña pieza $<1 \mathrm{~cm}^{3}$ del meteorito Heredia (de 2,9 g). Este pequeño espécimen (aprox. $5 \mathrm{~mm}$ x 8mm x 10 mm, Fig. 2) llegó a la NEMS probablemente adquirida del Prof. Terry Schmidt, quien laboraba en el Tiara Observatory, de donde pasó a la NEMS (R. Kempton, com. escrita, 2003), y de allí adquirido para una colección personal. Fue repatriado a Costa Rica en el 2002, y luego donado a finales de ese año a la Colección de Minerales y Rocas de la Escuela Centroamericana de Geología de la Universidad de Costa Rica, donde actualmente se exhibe (Soto, 2003; Figs. 2 y 3).

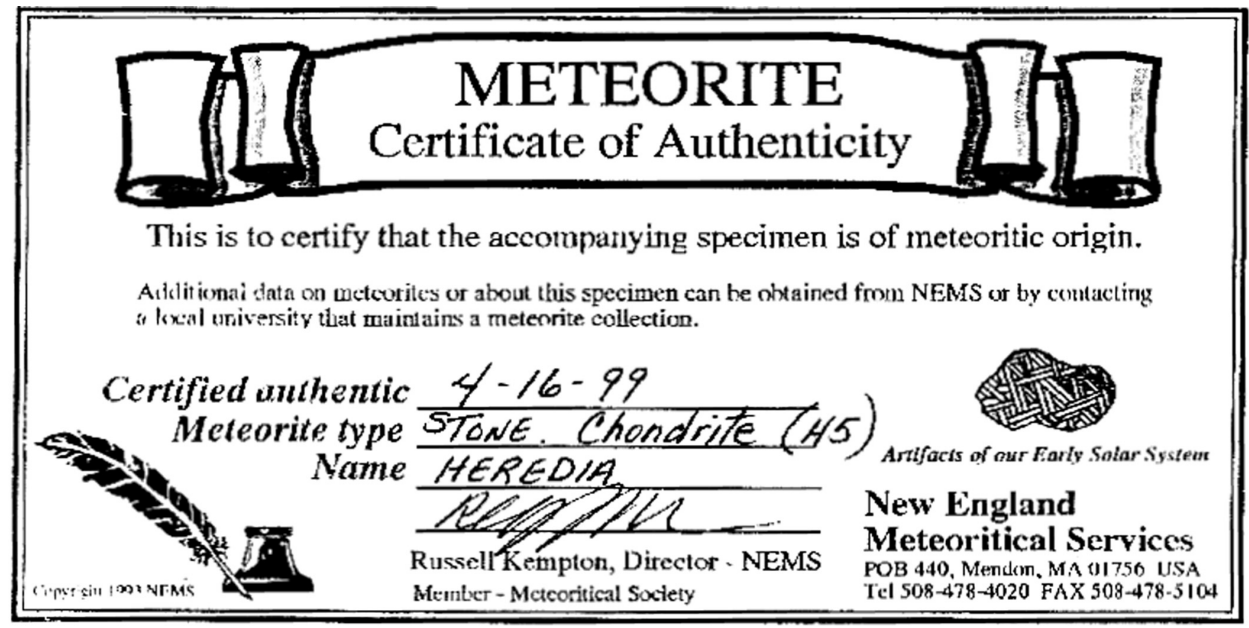

Fig. 3: Certificado de autenticidad del espécimen de la figura 2, expedido por la New England Meteoritical Services. 


\section{Datos hemerográficos de meteoros a princi- pios del siglo $\mathrm{XX}$}

Algunos diarios nacionales dieron numerosos informes de sismos o eventos astrofísicos en sus páginas entre finales del siglo XIX y principios del siglo XX (Peraldo, 2003). Hay una serie de reportes, sobre todo en El Noticiero, que se prestó a dar informaciones poco conocidas en otros medios, y que curiosamente no aparecen citadas en el libro de González (1910) ni en trabajos de otros estudiosos de la época, lo que en parte hace dudar de la total veracidad de las informaciones. Algunas de ellas, disponibles en las hemerotecas costarricenses, han sido recopiladas por G. Peraldo (UCR), quien amablemente ha cedido copias:

- El Noticiero del 13 de noviembre de 1903 da cuenta de "Un bólido el día 9 de noviembre, a las 10 h 23 m y dirección SSW.” No presenta más detalles ni localidades.

- El Noticiero del 2 de diciembre de 1905 refiere que "El lunes pasado [27 de noviembre], se vio en Barva un aerolito como a las 10 1/2 de la noche, se desprendió del oeste y corrió hacia el este. La bola de fuego como se le llamaba era del tamaño de una bola de fútbol, pasó a corta distancia del suelo como a $300 \mathrm{~m}$. y al apagarse se oyó una detonación sorda como de un cañonazo y enseguida como lámparas eléctricas que hayan hecho descarga."

- El Noticiero del 17 de mayo de 1906: “[El] 15, a las 10 h 19 m hubo un trayecto de un bólido con trayecto SSW - NNE por espacio de unos 10 seg." No menciona detalles sobre las localidades.

- La Prensa Libre del 7 de noviembre de 1907: "Anoche hubo una lluvia de exhalaciones estelares. Una observación cerca de las 9 de la noche y con dirección NO, 5 exhalaciones. Eran estas brillantes y desplegaban a su paso difusas bandas luminosas. Una persona Alvarado vio tres en el intervalo de 15 minutos caer en dirección SO. El tercer aerolito era mayor que sus dos compañeros y tenía un tinte rojo acentuado. Pocos segundos después de ocultar y en la dirección que lo hizo hubo algo así como un incendio que estalló iluminando el espacio."
- El Noticiero del 13 de noviembre de 1910: "El 10 a las 3 a.m. fue visto en el cielo una bola luminosa [que] alumbró el espacio como la de la noche del terremoto [ver el siguiente subcapítulo sobre la bola de fuego del 4 de mayo], haciendo un espantoso ruido. Este fenómeno fue presenciado por numerosas personas."

- El Noticiero del 6 de junio de 1912: "Desde Tortuguero 3 [de junio]: Los vecinos vieron desde la cumbre de un cerro, a eso de las 7 de la noche, [que] descendía paulatinamente una bola de fuego que despedía chispas. Una explosión parecida al estampido de un cañonazo. El aerolito se había estrellado contra un árbol de coposo ramaje. En el lugar del suceso se podía observar (minerales) como si hubieran estado en un horno de fundición."

Los primeros cinco reportes parecen coincidir con bolas de fuego, pero debido a que no hay mayores evidencias ni reportes de estudiosos, se tienen como dudosos, y solo destaca el de Barva, por dar detalles de localidades de observación. El sexto, califica igual, excepto que la descripción de que los fragmentos tenían el aspecto de trozos de fundición, coincide con las típicas cortezas de fusión de los meteoritos durante su trayecto en la atmósfera. Para tales épocas y nuestro entorno, estas rocas meteoríticas eran poco conocidas, lo que le da cierto grado de credibilidad al reporte, pero como no se conservaron fragmentos, ni nadie los estudió, se califica como dudoso.

\section{Una gran bola de fuego: 4 de mayo de 1910}

Don Anastasio Alfaro suscribió un informe del Museo Nacional sobre el terremoto de Cartago del 4 de mayo de 1910, publicado dentro de los anexos del libro de don Cleto (González, 1910). Cuenta don Anastasio que, por indicación del geólogo estadounidense Jaggar, se imprimieron unas hojas de cuestionarios sobre el terremoto que se distribuyeron por todo el país y de las cuales recibieron una cálida respuesta. Uno de los aspectos llamativos, aparte del terremoto como tal, lo anota don Anastasio como un aparte titulado "Iluminación posterior", que textualmente dice: 
"Hay igualmente gran número de observadores que aseguran haber visto un bólido, que pasó de E. á O. Sobre la cordillera volcánica, desde el Turrialba hasta atravesar la península de Nicoya, siguiendo, poco más o menos, el paralelo $10^{\circ}$ L.N. Con respecto á la hora, varía mucho pero todos están de acuerdo en que pasó rápidamente, poco después del terremoto, algunos estimaron el intervalo, entre uno y otro fenómeno, en segundos solamente, pero otros dicen que fué un cuarto de hora más tarde ó veinte minutos."

Algunos testigos presenciales, como la abuela materna de I. Boschini (com. oral, 1990), que vivía en Cartago cuando el terremoto, han referido la historia de la bola de fuego después del terremoto, con certera precisión y detalle. A pesar de que en varios terremotos se han observado fenómenos luminosos en el cielo, similares a "auroras boreales", de los cuales incluso hay testimonios objetivos de geólogos (P. Denyer, com. escrita, 2004), me parece que no cabe ninguna duda de la ocurrencia de la bola de fuego (y no simples "luminosidades"), pero esta no tiene ninguna relación con el terremoto, ni parece tenerla con el cometa Halley, que en ese momento se mostraba en los cielos terrestres (este evento astrofísico no se recaba en este trabajo debido a que es mundialmente conocido). Parece una casualidad impresionante, pero sí ocurrió el fenómeno del meteoro. En el caso de que hubiera sido acompañado de sonidos, no hay reportes, y si hubo un meteorito asociado, con seguridad cayó en el Océano Pacífico (Fig. 1). Este evento inspiró al célebre escritor Carlos Gagini, que incluyó una descripción literaria en su cuento "El Tesoro del Coco" (reproducido en una antología: Gagini, 1974): "Eran las seis y media de la tarde cuando divisamos la rada a donde nos dirigíamos. Pero en aquel instante el océano se agitó de una manera extraña y con rumor formidable, mientras un resplandor rojizo iluminó el cielo, un enorme globo de fuego surcó el firmamento y fue a sepultarse en las aguas del Golfo de Nicoya. Tan inexplicable fenómeno fue lo único que nos ocurrió durante la travesía”. Tal travesía, en su cuento, ocurría el 4 de mayo de 1910, a su regreso de haber desenterrado el tesoro de la Isla del Coco.

\section{CASOS ANECDÓTICOS}

\section{El meteorito férrico de San Pedro de Poás (1962?-63?)}

Con motivo del Ciclo de Conferencias Geológicas de Costa Rica, celebradas en San José en setiembre de 1980, se montó una exhibición de minerales y rocas en los ventanales de la Librería Universal, en la Avenida Central. Una de las muestras más llamativas era un "meteorito metálico", que muchos geólogos aún conservan en su mente, aunque no recuerdan su procedencia.

Mario Fernández Castro, geólogo y profesor en la Universidad de Costa Rica, contó la historia de este meteorito. Corría 1962 ó 1963, cuando él laboraba en la Dirección de Geología, Minas y Petróleo, en donde había un espacio físico para evacuar consultas de gente que llegara con preguntas sobre rocas y minerales o cualquier tópico afín. Dice don Mario que un día llegó un campesino con un saco de gangoche, en donde llevaba cuatro o cinco fragmentos de rocas muy pesadas. El campesino relató que una noche algunos días atrás, vio en un cerrito cerca de su casa en un cafetal de su propiedad en San Pedro de Poás (Fig. 1), algo así como un "juego de pólvora", súbito y fugaz. Al día siguiente se fue al cafetal a ver si encontraba algo, y en efecto, halló unas piedras negras que no había visto antes. Esas eran las rocas que había cargado en el saco de gangoche y que ahora le estaba enseñando. A don Mario le llamó la atención el aspecto denso de las rocas, con una parte de costra fundida y otra cristalizada. Pensó en un meteorito, y entonces la curiosidad le hizo preguntarle de manera emocionada al campesino, el dónde, el cómo y el cuándo. Esto hizo entrar en sospechas al visitante, y continuó con evasivas, de modo que no hubo forma de obtener más información. Le regaló una de las muestras de roca y se marchó. No se supo de él nunca más. Tenía pues, don Mario en sus manos, una muestra de un posible 
meteorito. Años después, se hizo un corte de la roca y se apreciaron estructuras de Widmanstätten, típicas de un meteorito férrico. Después de aquella exposición, se le devolvió la muestra a don Mario, quien como buen geólogo, la guardó en su colección. Al cabo de los años, sin embargo, la roca se ha extraviado y no se ha podido tener acceso a ella.

\section{La presunta bola de fuego y meteorito de Limón, 15 de abril de 1990}

El jueves 19 de abril de 1990 se recibió en la Sección de Sismología e Ingeniería Sísmica del ICE, un telefonema del geólogo Mario Fernández Arce (Universidad de Costa Rica) informando de una llamada que relataba la caída de una bola de fuego cerca de Limón, y la recuperación de un objeto que parecía ser el causante del fenómeno. El informante, Sr. Virgilio Vindas, funcionario de JAPDEVA, corroboró la información. Según el Sr. Vindas, tres guardas del muelle de JAPDEVA vieron caer, cerca de las 11:00 p.m. del domingo 15, una "bola de fuego encendida", con un zumbido, cerca del tajamar, unos 50-100 m, mar adentro.

El miércoles 18, cerca de las 17:30 horas, un buzo vio dentro del agua, en el punto de la caída del objeto mencionado, una "bola rojiza, brillante" que sacó del mar. Al contacto con la atmósfera, el objeto emanó gases que le quemaron el guante, por lo que lanzó el cuerpo extraído en un sitio entre el acceso al muelle y el tajamar, que es un relleno de detritos. En ese momento, el tamaño del cuerpo era como "el de una bola de fútbol" (unos $25 \mathrm{~cm}$ de diámetro) y continuó emanando gases nauseabundos (esto es: se estaba oxidando rápidamente), y quemó la vegetación y el suelo en un radio de pocos centímetros. El objeto fue interpretado por turistas que se encontraban en el muelle, como un meteorito. El Sr.Vindas oyó del asunto y acudió al sitio, donde logró rescatar un fragmento $\left(0,5 \mathrm{~cm}^{3}\right)$ antes de que el cuerpo se quemara totalmente. Según el Sr. Vindas, los gases eran densos, blancos, de olor "a quemado". Al raspar el objeto, "se quemaba con más facilidad" y daba la impresión de estar encendido.
El día 20, se examinó el resto recobrado, conservado dentro de una botella con agua. Tenía una consistencia sólida, no deformable, de color rosa-naranja, y al ser sacado del agua, tuvo una reacción exotérmica con desprendimiento de vapor blanco, de olor ácido, nauseabundo. Se descartó la posibilidad de que fuera un resto de satélite artificial o un meteorito. En el sitio de extracción se comprobó que el objeto quemó las plantas en unos decímetros a la redonda y calcinó parcialmente el relleno arcillo-arenoso que lo subyacía (dando un color pardo-naranja). Al remover el detrito, pequeñas partículas revueltas en él, reaccionaron con el aire, con las mismas características descritas y un sonido de ignición. Se tomó una muestra del detrito del relleno y se transportó a San José, junto con el residuo recuperado, para un análisis. Se comprobó la ausencia de radiactividad en el Laboratorio de Física Nuclear de la Universidad de Costa Rica (atención del físico nuclear MSc. Alfonso Salazar). Luego, el Dr. Julio Mata, de la Escuela de Química de la UCR, analizó la muestra y determinó que era fósforo de alta pureza.

El Prof. Mata, en una nota del 26 de abril de 1990, escribió sobre el análisis: “...me conduce a informar que se trata de fósforo elemental (variedad blanca). Ya que este elemento es constituyente de bombas incendiarias, y las extrañas referencias de los testigos en cuanto a su aparición, le sugiero que informe de este asunto al despacho del Ministro de Seguridad Pública [una copia de la nota fue dirigida al Ministro de ese ramo]."

El 5 de junio le envié una nota al Dr. Glenn J. MacPherson, curador asociado de la División de Meteoritos del Instituto Smithsoniano en Washington. En la carta le explicaba suscintamente el hecho, y le resaltaba que no era radiactivo y el análisis químico evidenciaba ser fósforo puro. Le hacía cuatro preguntas: 1- Si había algún meteorito o cuerpo cósmico conocido hecho de fósforo; 2- si había algún tipo de combustible para satélite artificial compuesto de fósforo; 3- si había alguna otra posibilidad que no fuera antropogénica para el componente de la bola de fuego, y 4- en el caso de que fuera material antropogénico, si él tenía alguna idea de qué podía ser. El 20 de junio, MacPherson contestó: 
"Ciertamente, el comportamiento del material al removerse del agua, es típico del fósforo blanco. No hay material conocido en la Tierra o el espacio que esté compuesto de fósforo elemental; el material encontrado en Costa Rica es casi ciertamente de origen humano. Es comúnmente usado por los militares, como balas trazadoras para seguir el campo de artillería; esto podría estar en entera consistencia con la observación de él cayendo a tierra. El fósforo se quema inmediatamente en contacto con el aire, y cuando es disparado de una pistola, deja una traza claramente visible tras él. Esto permite a los disparadores ver su objetivo y ajustarlo. Yo creo que este es el origen de esta 'bola de fuego'. El fósforo blanco es peligroso y venenoso. Usted debería presentar una queja ante las autoridades militares locales. Fue muy bueno que el buzo que la recogió [la muestra que estaba en el lecho marino] usaba guantes, porque produce quemaduras muy dolorosas que son lentas de sanar."

Por algún motivo desconocido, alguien disparó la bala de fósforo en Limón. Un caso de una "bola de fuego", pero no de carácter meteorítico, sino antropogénico.

\section{La bola de fuego del 15 de enero de 1991}

Una bola de fuego fue reportada como vista en Costa Rica, el 15 de enero de 1991, a las 03:03 a.m., en Miramar, observada por Elaine EureHenderson. Así fue reportada en el Boletín del Global Volcanism Network, que para esos años aún incluía información de meteoritos y meteoros en sus páginas (GVN, 1991). No existe ningún otro dato disponible, ni pude nunca, localizar a la observadora.

\section{La ¿bola de fuego? y ¿meteorito? de La Cruz, 23 de marzo de 1993}

El día 23 de marzo de 1993, cerca de las 00:50 de la madrugada, los señores Roberto Scott y José Santos Carrillo Castillo observaron una bola de fuego desde su automotor cerca de $5 \mathrm{~km}$ al sur de La Cruz, en la vecindad de la frontera con Nicaragua. El "meteoro" -según palabras del señor Scott- "era de color rojo brillante, del tamaño de una bola de basquetbol y tenía una estela o cola luminosa roja a su paso, con centro verdoso". La trayectoria era de $\mathrm{N}$ a $\mathrm{S}$, a "mediana velocidad", con un ángulo de caída de cerca de 15-20 con respecto a la horizontal. El Sr. Scott desaceleró el vehículo a unos $25 \mathrm{~km} / \mathrm{h}$ al observar el fenómeno, que se prolongó por 10-15 segundos. Luego, el "meteoro" fue observado caer a una distancia estimada de $1 \mathrm{a} 3 \mathrm{~km}$ al este de la carretera, en terrenos del Parque Nacional de Guanacaste, y vieron mantenerse durante varios segundos el resplandor en tierra, similar a una hoguera. Debido a que el vehículo aún estaba en marcha, no oyeron ningún sonido durante su paso, ni durante el impacto. El área del posible impacto del meteorito estaría entre las coordenadas $11^{\circ} 00,65^{\prime}$ $11^{\circ} 01,71^{\prime}$ latitud norte y $85^{\circ} 36,59^{\prime}-85^{\circ} 38,24^{\prime}$ longitud oeste (un área de unos $6 \mathrm{~km}^{2}$ ). Se consultó a guardas de un puesto de vigilancia distante $3 \mathrm{~km}$ del posible impacto, pero debido a la hora, no notaron nada anómalo. No se obtuvieron otros reportes de testigos del fenómeno.

El lugar del posible impacto fue visitado en compañía del geólogo Héctor Flores -con el apoyo de la Comisión Nacional de Emergencias-, el día 5 de abril. El terreno es parcialmente plano, pero cortado por valles profundos y abruptos, con vegetación de bosque seco, bajo y duro y pastos aislados. Debido a estas limitantes, y a la pobre definición del sitio exacto de impacto, este no fue encontrado. El hecho además, de su trayectoria desde el lado nicaragüense, y que hayan descrito "una hoguera" tras el impacto, no es típico de un meteorito, sino de algo antropogénico. Es solo una hipótesis plausible.

\section{Florencia, 14 de abril de 1994}

El día 16 de abril de 1994 el diario La Nación publicó el titular "Meteoro cayó en San Carlos" (pág. 16A). Esa misma semana, el semanario en inglés The Tico Times hizo eco de la noticia y la tituló más atractiva: "Meteorite Scares School Children" ["Meteorito asusta a niños escolares", pág. 7], específicamente en Florencia. 
La nota decía que un grupo de niños observó una bola de fuego rosada que se desprendió del cielo como a las 6:30 de la noche, perdió altura y cayó en un lote. Al chocar contra la tierra se fue derritiendo en forma circular, las llamas que provocó quemaron unos sesenta metros cuadrados, y unos diez minutos después, la bola se consumió. Literalmente atribuían el hecho a que "aparentemente, se trataba de un meteorito o estrella fugaz, un cuerpo sólido espacial que se desintegra al entrar en contacto con la atmósfera". Al leer lo de la quema del lote, entramos en sospechas, pues los meteoritos son fríos, y al ingresar en la atmósfera y quemarse por fricción, solo se funde una parte externa, pero el interior permanece frío. $\mathrm{Si}$ tocan tierra, llegan tibios o ligeramente calientes, pero no tanto para causar un fuego.

Procedimos (junto con G.E. Alvarado y Chico Arias) a visitar el sitio y comprobamos la noticia respecto a la quema del charral. Recabamos además testimonios y concluimos, de que no se trataba de un meteorito. Dos días después, se supo por los telenoticieros, que lo que había causado la bola de fuego, era una bengala de alto poder, disparada por un individuo no identificado. Otro caso de bolas de fuego causadas por trazadores bélicos o similares, como el de Limón y quizás el de La Cruz. Otro intento fallido por encontrar un meteorito.

\section{Lomas de Barbudal, 22 de abril de 1994}

A principios de mayo de 1994, el colega Guillermo E. Alvarado recibió una llamada telefónica de su amigo geógrafo Minor Moya, en la cual le contaba de una bola de fuego vista el día 22 de abril por dos vecinos de Lomas de Barbudal, unos $15 \mathrm{~km}$ al suroeste de Bagaces. Uno de ellos quedó encandilado por el suceso, que venía de norte a sur, pero no estaban seguros si hubo o no estruendo o si hubo un impacto. El sitio del posible impacto estaría entre las Lomas de Barbudal y la Hacienda "El Pelón de la Bajura". Guillermo se apersonó algunos días después, a buscar el sitio, con resultados infructuosos. No deja de resultar sospechoso que fuera otra bola de fuego, con un trazo de norte a sur, similar al de La Cruz.

\section{La supuesta explosión de un meteoro, 11 de julio de 1995}

Cerca de las 9 a.m. del 11 de julio de 1995, una explosión fue escuchada en varios lugares del Valle Central y el Pacífico central, lo que causó alarma, especialmente en los poblados de La Gloria de Puriscal y San Gabriel de Turrubares. La Guardia Rural de Turrubares contactó a la Red Sismológica Nacional (RSN: ICEUCR), quienes realizaron una inspección de campo y brindaron un informe (Rojas, 1995). No se debió a ningún sismo, ni a explosivos, ni a un percance aéreo. El informe desechó un impacto meteorítico debido a que "no se observaron evidencias claras de quemaduras en la vegetación u hojas de los árboles ni radiactividad". Como se discutió, sin embargo, los meteoritos llegan fríos y son incapaces de provocar quemaduras o incendios y menos radiactividad, porque no tienen contenidos altos de materiales radiactivos. No encontraron ningún impacto, tampoco. Lo que sí encontraron fue un deslizamiento de cerca de una hectárea en una ladera de la margen derecha del cañón del río Tulín. El informe sugirió "una posible detonación de un aerolito en las capas atmosféricas intermedias, sobre ese sector". Sin embargo, esta explicación tampoco parece plausible, pues si así hubiera sido, a pesar de la hora matutina, un evento de esta magnitud hubiera tenido, como característica más conspicua, la generación de una bola de fuego, que hubiera sido visible por un trayecto considerable, y este no fue el caso. Nadie vio nada. En mi opinión, el deslizamiento per se, súbito, debería ser la explicación más creíble del ruido escuchado, aunque por supuesto, no explicaría la enorme área donde se escuchó. Una incógnita más, pero no atribuible a meteoros o meteoritos.

\section{La bola de fuego del 12 de junio de 1999}

El Diario Extra del 16 de junio de 1999 publicó un artículo (págs. 16-17) titulado "Vecinos de Punta Mala ratifican las versiones sobre presunto meteorito", da cuenta de una bola de fuego que cruzó el cielo desde el mar hacia el 
continente, cerca de la medianoche. El poblado queda en la desembocadura del río Grande de Térraba, y la descripción es de una bola de fuego "como un bombillo gigante, el cual salió del mar por detrás de la isla Garza [una de las islas del estuario del río] y cruzó el espacio para luego caer detrás de la montaña." El color era amarillo y dejó una estela blanca que desapareció mientras la bola caía tras la montaña de la Fila Costeña, con "un estruendo como la explosión de una carga de dinamita". La descripción es típica de un meteoro que provoca una bola de fuego. El fuerte sonido no necesariamente implica un impacto, sino que podría ser la fragmentación del meteoro en la atmósfera alta. Si cayó un meteorito, éste debe haberse estrellado en algún lugar montañoso de las filas Alta, Marítima o Margarita, donde es improbable encontrar el sitio de su llegada a tierra. Sin embargo, por las características acotadas por los numerosos testigos del fenómeno, estoy de acuerdo en calificarlo como una auténtica bola de fuego.

\section{PROBABILIDADES DE QUE CAIGA UN METEORITO EN COSTA RICA}

Como se expuso atrás, la caída de meteoritos de tamaños encontrables, es en realidad un evento raro, y solo los micrometeoritos son muy frecuentes, pero de tan ínfimo tamaño, que pasan inadvertidos en general. Durante las primeras centenas de millones de años de historia del Sistema Solar, la tasa de bombardeo de meteoritos no solo era más alta, sino que las dimensiones de los bólidos eran enormes, y aun de vez en cuando en la historia geológica terrestre ocurren grandes impactos (véase por ejemplo Grieve, 1988; 1994). Conforme ha pasado el tiempo, la cantidad y tamaño de meteoritos ha ido disminuyendo. Necesitamos además que los meteoritos que vagan fuera del cinturón principal de asteroides tengan rutas de colisión posible con la Tierra, las cuales son caóticas (Wisdom, 1985). Y aun así, muchos de esos meteoritos acaban consumiéndose durante el vuelo en la atmósfera terrestre (entre el $30 \%$ y 60\%). Con base en cálculos hechos por Halliday et al. (1989), que mantuvieron una red de observación de bolas de fuego en Canadá durante once años, y haciendo las extrapolaciones para Costa Rica, asumiendo una tasa de caída similar en todas las latitudes de la Tierra (cosa que no es completamente cierto, porque la teoría predice que por cuatro caídas de meteoritos en el ecuador solo habrían tres en los polos: Hutchison \& Graham, 1993), podemos decir que las probabilidades de que caiga un meteorito en Costa Rica, con un peso de al menos $1 \mathrm{~kg}$ es de 0,44/año, número que pareciera muy alto. Son probabilidades de que caiga, pero de eso a que se observe y se recupere, marca un trecho muy grande. Según el ejemplo comparativo citado de China, sería esperable recuperar un meteorito en Costa Rica cada 1200 años. Si tomamos como comparación la década de los noventa del siglo pasado, cuando tenemos una cobertura de población en casi todo el territorio nacional y medios de comunicación eficientes, notamos solo dos eventos observados de bolas de fuego (enero 1991 y junio 1999; Fig. 1), que nos da un promedio de una cada 5 años. Al menos el orden de los números de probabilidades y de observaciones coinciden (4,4 probables contra 2 observados). Por otra parte, se ha visto un meteorito caer, ser recuperado y conservado en siglo y medio, y a lo sumo dos más, de los que no se conserva prueba fehaciente, lo cual podría ser un número relativamente alto, comparado con lo esperable.

\section{DISPUTAS POR PROPIEDAD}

Me he preguntado varias veces sobre los derechos de propiedad de un meteorito que caiga en Costa Rica. Un meteorito es una roca y como tal, está compuesta de minerales. El Diccionario de Términos Geológicos del Instituto Geológico Estadounidense (Bates \& Jackson, Eds., 1984) define un depósito mineral como "una masa de material mineral que ocurre naturalmente,... usualmente de valor económico, sin distingo de su modo de origen". Parece lógico, en mi opinión, que entonces la "explotación" de un meteorito se rija por los principios de la Ley $\mathrm{N}^{\mathrm{o}} 6797$, el Código de Minería (1982), y el Decreto $\mathrm{N}^{\circ}$ 29300-MINAE, Reglamento al Código de 
Minería (2001) de los cuales se transcriben algunos artículos y notas, para discutir sus alcances, que por supuesto son interpretativos, pues no hay referencias explícitas a meteoritos.

El artículo 1 del Código dice que "El Estado tiene el dominio absoluto, inalienable e imprescriptible de todos los recursos minerales que existen en el territorio nacional y en su mar patrimonial, cualquiera que sea el origen, estado físico o naturaleza de las sustancias que contengan... el Estado podrá otorgar concesiones para el reconocimiento, exploración, explotación y beneficio de los recursos minerales, conforme con la presente ley." El Reglamento no define "recurso mineral", pero sí "recurso indicado" en el acápite 44) del artículo $4^{\circ}$ : "El que se estima que representa un interés económico intrínseco sobre la base de una exploración general que confirme las principales características geológicas de un yacimiento y que suministre una estimación inicial de sus dimensiones, forma, estructura y contenido".

Luego, el artículo 27 del Código escribe: "El respectivo Ministerio podrá otorgar directamente una concesión de explotación, sin necesidad de exigir el cumplimiento previo de la etapa de exploración, cuando los minerales estén a la vista o sea evidente su existencia, previa elaboración y aprobación del correspondiente proyecto de explotación”. Explotación está definido en el acápite 21) del artículo $4^{\circ}$ del Reglamento: "Extracción de minerales de un yacimiento de acuerdo a técnicas mineras de superficie o subterráneas". Además, el artículo 39 del Código: "Los yacimientos de placer ubicados en terrenos baldíos o en el lecho mismo de un río o quebrada, sobre los cuales no hubieran derechos mineros previos, podrán ser aprovechados libremente, siempre que el lavado se efectúe a mano." Y el artículo 41 del Código: "Cuando un yacimiento de placer se encuentre en un terreno cercado y éste sea de dominio privado, el propietario será el que tenga prioridad para efectuar los trabajos de aprovechamiento, pero deberá reconocer un porcentaje de la explotación del yacimiento a quien lo hubiese descubierto; tal reconocimiento se hará de acuerdo con el estudio técnico que deberá realizar la Dirección de Geología, Minas e Hidrocarburos."
A pesar de que un meteorito es de procedencia extraterrestre, es un recurso mineral que tiene un valor económico de mercado definido internacionalmente según su tipo, y si cayó en el pasado, o si cae en algún momento, se encuentra en el territorio nacional. Por tanto, según el artículo primero transcrito, independientemente de su origen o naturaleza, el Estado tiene dominio sobre él, pero no su propiedad. Aunque estrictamente hablando, un meteorito no es un yacimiento de placer, se comporta como tal: está suelto y a la vista, parcialmente enterrado, a lo sumo. Han de tomar en cuenta no sólo los pequeños meteoritos de algunos g ó kg de peso, que pueden ser una o varias piezas, sino la posibilidad de que caiga algún día un meteorito grande, que se fragmente y forme todo un campo de caída, con especímenes de gran tonelaje. En el caso de los especímenes pequeños, se puede aplicar el artículo 39, de modo que basta tomarlos con la mano, y pueden ser aprovechados libremente por su descubridor, siempre que sea en terrenos baldíos, o bien en propiedad privada, por su dueño. En caso de ser piezas de gran tamaño, o múltiples, podrá el dueño explotarlo con base en el artículo 41. Pero si algún científico, o cualquier otra persona lo descubren, podrá hacer uso de este mismo artículo para exigir su parte. A lo sumo, necesitarían cumplir el artículo 27. Es solo mi interpretación, pues no hay precedentes en Costa Rica. Cito un antecedente histórico en el extranjero: En Estados Unidos, debido a un sonado caso ocurrido en 1902, cuando un individuo descubrió y extrajo secretamente un meteorito de $12700 \mathrm{~kg}$ de una propiedad, pero luego fue demandado por los dueños del terreno para reposeer la roca, existe el precedente legal de que un meteorito pertenece al dueño del terreno sobre el cual cayó (McSween, 1999; pág. 10).

\section{SEGUROS CONTRA IMPACTOS DE METEORITOS}

Los meteoritos que caen en áreas pobladas son pocos. Sólo hay dos casos documentados de personas que hayan sido heridas por meteoritos al caer: en Japón en 1927 y en el estado de 
Alabama, EE.UU., en 1954. Este último caso es el mejor documentado: un fragmento de un meteorito pétreo de $3,9 \mathrm{~kg}$ atravesó el techo y el cielo raso de una casa, golpeó una radio grande e hirió a una mujer que estaba recostada en un sofá, quien sufrió heridas en su cadera y abdomen (Swindel \& Jones, 1956). Otro caso curioso es el del meteorito Nakhla, en Egipto en 1911, que mató a un perro. Como sólo son casos aislados reportados en el mundo, se puede afirmar que el hecho de ser golpeado por un meteorito es extremadamente raro. Con base en los cálculos que hicieron Halliday et al. (1985), extrapolados a Costa Rica, podríamos decir que las probabilidades de ser alcanzado por un meteorito es de una persona cada 11 mil años. A pesar de que los meteoritos pueden caer a cualquier hora, debido a la dirección de rotación terrestre y de órbita de los meteoros, la mayoría caen entre mediodía y medianoche (Hutchison \& Graham, 1993).

Para tomar un ejemplo de un país extenso, bien observado y extensamente poblado, tenemos que entre 1965 y 1992 se reportaron 19 recuperaciones de meteoritos caídos en Estados Unidos. De ellos, 8 causaron algún daño a edificios. Uno de los más insólitos fue el caso del 9 de octubre de 1992, a las 7:50 p.m., cuando un meteorito de $12 \mathrm{~kg}$ cayó sobre un carro parqueado en el poblado de Peekskill, estado de Nueva York. Al oír un fuerte ruido, el dueño del vehículo y un vecino descubrieron la roca, del tamaño de un balón de fútbol, que había entrado por el baúl del carro y estaba indentada a la calle. Previamente, una intensa bola de fuego fue avistada en varios estados, precisamente a la hora del fútbol estadounidense universitario, y por eso se convirtió en la bola de fuego más filmada de la historia. Afortunadamente para el dueño del vehículo, tanto el carro como el meteorito fueron adquiridos por el Museo Estadounidense de Historia Natural de Nueva Cork (GVN, 1992; Norton, 2002). Una vez más, con base en la extrapolación de datos de Halliday et al. (1985), para Costa Rica, tendríamos que las posibildades de tener daños en un edificio, es de uno cada 78 años.

Ante estos hechos y números de probabilidades, aun tan bajos, ¿habrá alguna manera de asegurarse en Costa Rica contra los meteoritos? A cualquier costarricense, su seguro social lo cubrirá, por ser enfermedad, invalidez o muerte, según sea el caso, puesto que es un accidente, sólo que de índole cósmico-terrestre. Los seguros de vida deberían pagar si alguien muere, pues es un accidente.

¿Qué sucede con bienes como vehículos automotores y edificios? En el caso de automóviles (INS, 2004a) la "Cobertura 'H' riesgos adicionales", a pesar de que no explicita un evento tal (ni erupciones volcánicas), sí incluye eventos naturales como temblor, terremoto, deslizamiento, derrumbe y rayo. Podría cubrir los daños, puesto que es un accidente causado por un objeto extraño, tal cual si fuera una roca desprendida de un paredón de la carretera, o la caída de un avión o un objeto desde él. En el caso de construcciones inmuebles (INS, 2004b), tampoco está contemplado explícitamente, pero las "coberturas" incluyen prácticamente cualquier daño causado por un evento natural, como rayo, caída de árboles, vientos huracanados, huracán o ciclón, inundación y deslizamiento, temblor y terremoto, erupción volcánica y maremoto. ¿Si una casa asegurada es impactada por un meteorito, el seguro debería pagar los daños? De cualquier manera, al reclamar los pagos en la denuncia respectiva ante el Instituto Nacional de Seguros, debe especificarse muy bien el caso, y será el INS quien finalmente decida, luego de la evaluación de un perito. Puesto que el impacto de aerolitos sucede contra casas y autos, aunque con muy bajas probabilidades, este ítem no necesariamente debería estar escrito explícitamente en las coberturas de seguros, pero sí ser considerado al pagarlo como un accidente.

\section{EPÍLOGO}

No es mucho lo que se aporta al conocimiento de los meteoritos en Costa Rica. Lo que se sabía del meteorito Heredia, solo es acrecentado por la presencia de un minúsculo fragmento en las colecciones nacionales. De los otros dos posibles meteoritos quizás nunca se vean muestras que prueben, sin duda alguna, su existencia. De las bolas de fuego, solo queda el testimonio escrito -no gráfico-, que puede ser interpretado 
de una u otra manera. Quede, sin embargo, el germen de buscar y talvez encontrar un meteorito desconocido. Improbable, mas no imposible. En el futuro, caerán. Me avisan.

\section{AGRADECIMIENTOS}

A Monica Grady (Museo de Historia Natural de Londres) y Russ Kempton (NEMS), por la útil información respecto al meteorito Heredia. A Gregorio Escalante, por sus datos y discusiones. A Mario Fernández Castro por compartir su historia. A Guillermo E. Alvarado (ICE), por cooperar con ciertas búsquedas a las que pocos se hubieran atrevido, cooperar con material bibliográfico y sostener discusiones con principio y final. A "Chopo" Flores y Luis Diego Morales (CNE) por tratar de encontrar lo inencontrable. A Percy Denyer (UCR) por revisar el texto, sus invaluables y críticas sugerencias, obtener un documento extraviado y la foto del minúsculo espécimen de Heredia. A Giovanni Peraldo (UCR) por proveer material hemerográfico difícil de obtener, y sus siempre estimulantes discusiones, compartiendo su erudición. A Siegfried Kussmaul (UCR) por su revisión enriquecedora del texto final. A Linda Sjöbohm por su ayuda en la parte gráfica y por su criticismo al escrito. Al Instituto Costarricense de Electricidad por facilitar búsquedas de posibles meteoritos. A Luis A. Madrigal (ICE) por contribuir en la investigación de material en la hemeroteca. A Fernando Alvarado (ICE) por realizar algunos comentarios al capítulo de "Disputas por propiedad". A las otras personas mencionadas en el texto por sus nombres, que con sus observaciones han hecho posible conocer más sobre los meteoros en Costa Rica, aunque no fueran meteoritos los que cayeran.

\section{REFERENCIAS}

Alfaro, A., 1924: El "Volcán Viejo". - Rev. Costa Rica, V: 130-133, San José.

Alvarado, G., 1989: Los volcanes de Costa Rica. - 175 págs. EUNED, San José.
Álvarez, L.W., Álvarez, W., Asaro, F. \& Michel, H.V., 1980: Extraterrestrial cause for the CretaceousTertiary extinction. - Science, 208: 1095-1108.

BATES, R.L. \& JACKSON, J.A. (eds.), 1984: Dictionary of Geological Terms [3 ${ }^{\mathrm{a}}$ ed.]. -571 págs. Preparado por The American Geological Institute, Anchor Press/ Doubleday, Nueva York.

BÜChNER, O., 1863: Die Meteoriten in Samml. - Leipzig, pág. 93.

Código De Minería, 1982: Ley No 6797 del 4 de octubre de 1982. - La Gaceta, 203, 22 de octubre de 1982.

Dengo, G. \& Escalante, G., 1980: Mapa preliminar de reconocimiento geológico y fotogeológico. Zona de influencia de la cuenca del río Savegre. - Escala 1:50 000, Instituto Costarricense de Electricidad, 1 hoja inédita, San José, Costa Rica.

Domeyko, I., 1859: Meteorito de Costa Rica. - An. Univ. Chile, 16: 235, Santiago.

FRENCH, B.M., 1990: 25 Years of the Impact-Volcanic Controversy. Is There Anything New Under the Sun or Inside the Earth? - EOS, Transactions, AGU, 71(17): 411-414.

GaginI, C., 1974: El Tesoro del Coco. - En: Autores costarricenses, Editorial Fernández Arce, 203-206.

Graham, A.L., Bevan, A.W.R. \& Hutchison, R., 1985: Catalogue of Meteorites [ $4^{\mathrm{a}}$ ed.]. - British Museum (Natural History), London, and University of Arizona, Tucson.

GonZÁLEZ, C., 1910: Temblores, terremotos, inundaciones y erupciones volcánicas en Costa Rica 1608-1910. - iv + 200 págs. Tipografía de Avelino Alsina, San José, Costa Rica.

GRIEVE, R.A.F., 1988: Terrestrial impact craters: their recognition nature and effects. - Rev. Geofís. 28: 145-178.

GRIEVE, R.A.F., 1994: Impact: a natural hazard in planetary evolution. - Episodes, 17(1-2): 9-17.

GVN, 1991: Fireballs. - Bull. Global Volcanism Network, 16(1): 14.

GVN, 1992: Meteorite fall. - Bull. Global Volcanism Network, 17(9): 17-18.

Halliday, I., Blackwell, A.T. \& Griffin. A.A., 1985: Meteorite impacts on humans and on buildings. Nature, 318(6044): 317. 
Halliday, I., Blackwell, A.T. \& Griffin. A.A., 1989: The flux of meteorites on the Earth's surface. - Meteoritics, 24: $173-178$.

Hildebrand, A.R., Penfield, G.T., Kring, D.A., Pilkington, M., Camargo, A., Jacobsen, S.B. \& Boynton, W.V., 1991: Chicxulub crater: A possible Cretaceous/Tertiary boundary impact crater on the Yucatán peninsula, Mexico. - Geology, 19: 867-871.

Humboldt, A. De, 1844: Cosmos. A Sketch of a Physical Description of the Universe. Vol. 1 [Traducido del alemán por E.C. Otté, 1901]. - 369 págs + notas (18 págs.) George Bell \& Sons, Londres.

Humboldt, A. DE, 1941: Viaje a las regiones equinocciales del Nuevo Continente. Hecho en 1799, 1800, 1801, 1802, 1803 y 1804 por A. de Humboldt y A. Bonpland, redactado por Libros $3^{\circ}$ y $4^{\circ}$. Tomo II. - 458 págs. Biblioteca Venezolana de Cultura, Caracas.

Hutchison, R., 1992: Earliest planetary melting -the view from meteorites. - En: CoX, K.G. \& BAKER, P.E. (Ed.): Essays on Magmas and other Earth Fluids (a Volume in Appreciation of Professor P.G. Harris). - J. Volcanol. Geotherm. Res. 50: 7-16.

Hutchison, R. \& Graham, A., 1993: Meteorites. - 60 págs. Sterling Publishing Company, Nueva York.

Instituto Nacional de Seguros, 2004a: Seguro Voluntario de Automóviles. Condiciones Generales. - 42 págs. Folleto público.

Instituto Nacional de Seguros, 2004b: Hogar Seguro 2000. - Folleto público, 1 hoja desplegable.

Lin, W., OuYANG, Z. \& WANG, S., 1995: Cosmochemistry in China. - Episodes, 18(1-2): 95-97.

Littmann, M., 1998: The Heavens on Fire: The Great Leonid Meteor Storms. - 348 págs. Cambridge University Press, Cambridge.

Mason, B., 1962: Meteorites. - xii + 274 págs. John Wiley \& Sons, Nueva York.

MAson, B., 1963: Olivine composition in chondrites. Geochim. Cosmochim. Acta, 27: 1011-1023.
McSweEn, H.Y. JR., 1999: Meteorites and Their Parent Planets [2 $2^{\mathrm{a}}$ ed.]. - 310 págs. Cambridge University Press, Cambridge.

Norton, O.R., 2002: The Cambridge Encyclopedia of Meteorites. - 354 págs. Cambridge University Press, Cambridge.

Peraldo, G., 2003: El pensamiento científico del siglo XIX y la evolución de la geología en Costa Rica y su enseñanza. - En: Peraldo, G. (Comp.): Ciencia y Técnica en la Costa Rica del siglo XIX. - Editorial Tecnológica de Costa Rica, págs. 37-86.

Reglamento Al Código De MineRíA, 2001: Decreto Nº 29300MINAE del 8 de febrero del 2001. - La Gaceta, 54, 16 de marzo del 2001.

RoJAs, W., 1995: Informe sobre la causa posible de una fuerte explosión escuchada al oeste del Valle Central y en la zona Pacífico Central de Costa Rica. - Red Sismológica Nacional, informe de prensa, 1 pág.

SAlguero, M., 1978: Un aerolito formó la Laguna de Río Cuarto. - Gentes y Paisajes, N 47, VII-78, p. 17B.

Schultz, L. \& KRuSE, H., 1983: He, Ne and Ar in meteorites: a data compilation. - Mainz.

Soto, G., 1989a: Heredia: un aerolito que nos cayó en 1857. - Bol. Informativo Colegio de Geólogos de Costa Rica, 2(1): 2.

Sото, G., 1989b: El meteorito tico. - Semanario Universidad, 861: págs. 6 y 16.

Soto, G.J., 2003: El regreso del meteorito Heredia. Seminario Universidad, 1512: pág. 2.

STRAUCH: W., 1997: Meteorito de la noche del Sábado, 26 de abril, 1997. - En: Sismos de Nicaragua (Bol. Sismol.), Abril, 1997: 6-7.

SWINDEL, G.W. \& JONES, W.B., 1956: The Sylacauga, Talladega County, Alabama aerolite: a recent meteoritic fall that injured a human. - Meteoritics, 1: 125-132.

WISDOM, J., 1985: Meteorites may follow a chaotic route to Earth. - Nature, 325(6022): 731-733. 